\title{
Wavelet Transform based Estimation of Images using different Thresholding Techniques
}

\author{
Sk. Ayesha \\ M.Tech Student \\ Dept.of ECE \\ Amara Institute of Engineering \& \\ Technology, Satulur
}

\author{
M. V. R. Amara Lingeswara \\ Rao \\ Assistant Professor \\ Dept.of ECE \\ Amara Institute of Engineering \& \\ Technology, Satulur
}

\author{
Koteswararao Mallaparapu \\ Assistant Professor \\ Dept.of ECE \\ Chalapathi Institute of Engineering \\ \& Technology, Lam
}

\begin{abstract}
Estimating the images using decimated wavelet transform is very popular technique in different applications. In this paper a new thresholding function with combination of Smoothly Clipped Absolute Deviation (SCAD), Hard thresholding and soft thresholding functions are introduced for wavelet based denoising of images. The proposed technique is applied for denoising of noisy images contaminated with additive white Gaussian noise using Top rule and Visu rule. The results are compared with that of existing SCAD, hard and soft functions denoising method. Root Mean Square Error (RMSE) and Peak Signal to Noise Ratio (PSNR) are used as parameters for testing the quality of denoising.
\end{abstract}

\section{Keywords}

Wavelet transform, decimated wavelet transform, image denoising, new thresholding function, top rule, Visu rule.

\section{INTRODUCTION}

Images are acquired in many applications like satellite communication, medical diagnosis. They will be transmitted from one place to another place. In this process noise will be added to the image. This noise affects the clarity of the image at the receiver. To get the original image, denoising of the image is required. Wavelet transform, due to its excellent localization property, has rapidly become an essential signal and image processing tool for a variety of applications, including denoising and compression. Wavelet denoising attempts to remove the noise present in the signal while maintaining the signal characteristics, regardless of its frequency content. Different methods are used for image denoising. The popular techniques for denoising of images are based on wavelet transform. Out of these methods, denoising of image using thresholding method is widely used technique. This method is proposed in this project. In this method wavelet transform is applied on the noisy image. The outputs of wavelet transform are the noisy wavelet coefficients. Then the threshold value is fixed by applying thresholding rule. The wavelet coefficients are changed using the threshold function. The output of the function is applied to the inverse wavelet transform. It gives the de-noised image. This method is implemented with different noise levels, different images, and different wavelets. These results are compared across the different methods.

\section{IMAGE DENOSING}

In the field of image processing major problem occurred with noisy image. Image denoising is to recover an original image from an observed noisy image. The wavelet thresholding for image de-noising involves the following steps

1. To get the wavelet coefficients by using the wavelet decomposition on the input noisy image.

$$
\mathrm{p}=\mathrm{P}(\mathrm{B}+\mathrm{N})
$$

Where $\mathrm{p}$ is the wavelet coefficients, $\mathrm{P}$ is the wavelet transform, $\mathrm{N}$ is the noise data, and $\mathrm{B}$ is the original image.

2. Apply the thresholding function by using the thresholding rules, on the wavelet co- efficient.

$$
\mathrm{P}^{\prime}=\delta(\mathrm{p})
$$

Where $\mathrm{P}^{\prime}$ is the optimal estimation of the wavelet coefficients, $\delta(p)$ wavelet thresholding function , $\square$ is threshold.

3. Then apply the inverse wavelet transform on the modified wavelet coefficients and get the de-noised image

$$
\mathrm{B}^{\prime}=\mathrm{P}-1 \mathrm{p}^{\prime}
$$

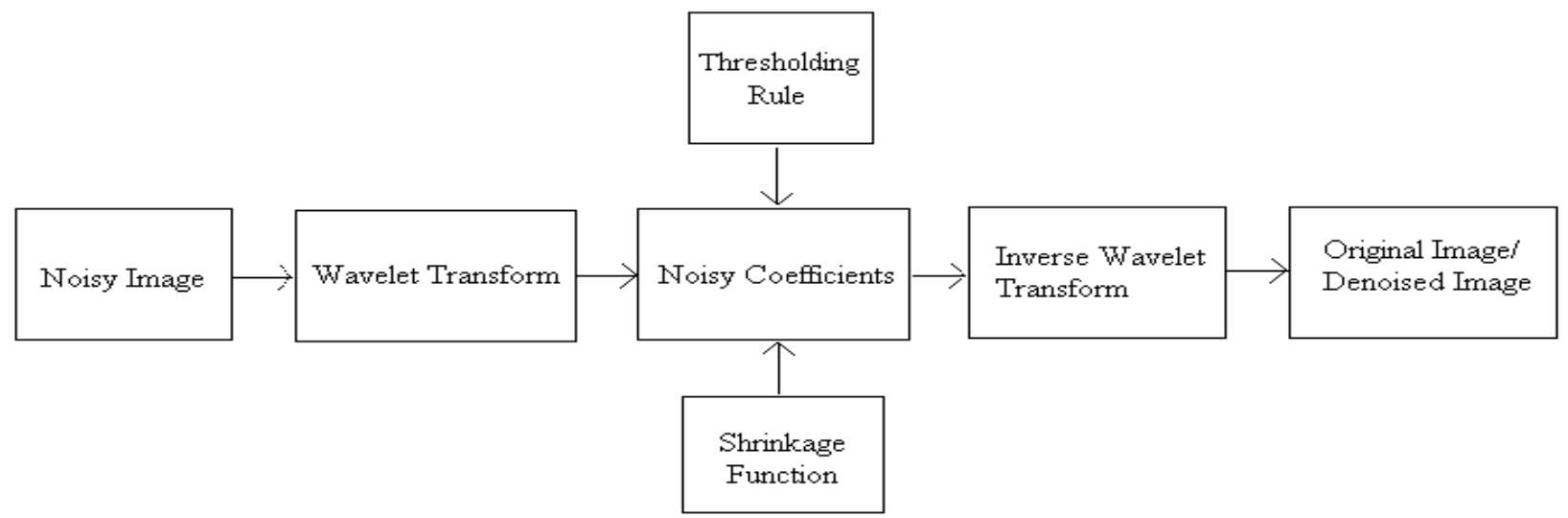

Fig 4.1: Block Diagram 


\section{THRESHOLDING RULES}

\subsection{Top Rule}

Top rule is a global thresholding method and it is independent of thresholding function selected. Given $\mathrm{Z}$ as the fraction of the largest coefficients to keep, the threshold is set to be the (1-Z) th quintile of empirical distribution of absolute values of wavelet coefficients.

\subsection{Visu Rule}

This rule was developed by donoho and Johnston. This is a universal thresholding method not depending on thresholding filter selected. The threshold value is calculated by

$$
\lambda=\sigma \sqrt{2 \log L}
$$

where $L$ is length of the image, $\sigma$ is the standard deviation, and $\lambda$ is the threshold value.

\section{THRESHOLDING FUNCTIONS}

Thresholding is a simple non-linear technique, which operates on one wavelet coefficient at a time. In its most basic form, each coefficient is threshold by comparing against threshold. If the coefficient is smaller than threshold then it is set to be zero otherwise it is kept or modified. Then inverse wavelet transform will be applied on the result which leads to reconstruction with the essential signal characteristics and with less noise. Since the work of Donoho and Johnstone, there has been much research on finding thresholds, however few are specifically designed for images.

\subsection{Soft thresholding function}

If the coefficient value (p) is greater than the threshold value $(\lambda)$, then it taken as the difference between coefficient value and threshold value otherwise the value is zero.

$S(p, \lambda)=\left\{\begin{array}{c}\operatorname{sgn}(p)(|p|-|\lambda|) \text { for }|p|>\lambda \\ 0 \text { otherwise }\end{array}\right.$

\subsection{SCAD function}

$$
\operatorname{SCAD}(p, \lambda)=\left\{\begin{array}{c}
\operatorname{sign}(p) \max (0,|p|-\lambda) \\
\text { if }|p| \leq 2 \lambda \\
(\alpha-1) p-\alpha \lambda \operatorname{sign}(p) \\
\text { if } 2 \lambda \leq|p|<\alpha \lambda \\
p \quad \text { if }|p|>\alpha \lambda
\end{array}\right.
$$

Where $\alpha=3.7$, SCAD means smoothly clipped absolute deviation.

\subsection{Hard thresholding function}

Donoho and Johnston proposed hard thresholding filter. A threshold value is selected by adopting some sort of threshold rules. Hard thresholding sets any coefficient below or equal to the threshold to zero. The coefficients above this threshold value are retained. The hard-thresholding " $\mathrm{H}(\mathrm{e}, \lambda)$ " is denoted by

$$
\begin{aligned}
\mathrm{H}(\mathrm{e}, \lambda) & =\mathrm{e} \text { for }|\mathrm{e}|>\lambda \\
& =0 \text { otherwise }
\end{aligned}
$$

\subsection{New thresholding function}

It is developed based on the median of the SCAD function, hard function and soft thresholding function. If the coefficient value $(p)$ is greater than the thresholding value $(\lambda)$, then it allows the function value, otherwise it's taken the twenty percentage of coefficient value with output of function.

\section{EXPERIMENTAL RESULTS AND DISCUSSION}

The performance of the proposed thresholding function is evaluated and compared with soft hard and SCAD thresholding functions using wavelets. The experiment is conducted on the image 512 X 512 of size. The Gaussian noise with different variations added to the input image. By applying the wavelets transforms to get the wavelet coefficients those coefficients are modified according in to the shrinkage function and apply the inverse wavelet transforms on the image finally get the original image or denoising image. The performance of the denoising is evaluated by using the PSNR \& RMSE.

RMSE is defined as root mean squared error between original image and denoising image

$\operatorname{RMSE}=\sqrt{\frac{1}{n} \sum_{i=1}^{n}(X(i)-\hat{X}(i))^{2}}$

PSNR $=20 \log 10(255 /$ square root of MSE $)$

Where $X(i)$ the original image is $\hat{X}(i)$ is the de-noised image, $\mathrm{n}$ is number of samples. The simulation experiment is repeated 100 times and average values are taken the process is conducted on different images and the results are same. This simulation is developed in MATLAB environment. The results of images for $\sigma=10,20$ and 30 using Soft,hard, SCAD and new thresholding filter with visu rule method and top rule method are shown in Table 1 and Table2. The original and denoised images using new thresholding filter with visu rule method and Top rule method are shown in Figs 1-4. Graph 14 shows the comparison of the results of visu rule and top rule methods.

For $\sigma=10$, RMSE is 10.8689 and PSNR is 27.4071 are obtained on denoising of noisy image with SCAD filter and RMSE is 12.8571 and PSNR is 25.9480 are obtained with soft thresholding function and RMSE is 8.0409 and PSNR is 30.0247 are obtained with hard thresholding function using visu rule method (Table1). For new thresholding function RMSE of 8.8139 and PSNR of 29.2275 are obtained (Table 1). From the above values we can observe the new thresholding function performs better than the SCAD and soft thresholding function. Similarly performance is obtained for the remaining values $\sigma=20$ and 30 (Table 1 ).

Using Top Rule method, for $\sigma=10$, RMSE of 8.6454 and PSNR of 32.3137 are obtained on denoising of noisy image with SCAD thresholding filter and RMSE of 7.9451 and PSNR of 30.1288 are obtained with soft thresholding filter and RMSE of 8.8571 and PSNR of 29.1850 are obtained with hard thresholding filter. For new thresholding filter, RMSE of 7.2633 and PSNR of 30.9081 are obtained (Table 2). This indicates that the new thresholding filter performs better than both SCAD, hard and soft thresholding filters. For $\sigma=20$ and 30 the new thresholding function gives better results than the SCAD, hard functions only. 
Table 1: Denoising Results of Fingerprint image using SCAD, hard, Soft and new Thresholding Function: Visu rule.

\begin{tabular}{|l|c|c|c|c|c|c|}
\hline & \multicolumn{2}{|c|}{$\sigma=10$} & \multicolumn{2}{c|}{$\sigma=20$} & \multicolumn{2}{c|}{$\sigma=30$} \\
\hline & RMSE & PSNR & RMSE & PSNR & RMSE & PSNR \\
\hline Noisy Image & 9.9616 & 28.1642 & 19.9724 & 22.1222 & 29.9724 & 18.5964 \\
\hline Estimation using Soft function & 12.8571 & 25.9480 & 17.6203 & 23.2106 & 18.3817 & 22.8431 \\
\hline Estimation using SCAD function & 10.8689 & 27.4071 & 16.4470 & 23.8091 & 18.4187 & 22.8256 \\
\hline Estimation using Hard function & 8.0409 & 30.0247 & 11.3460 & 27.0339 & 15.8616 & 24.1238 \\
\hline $\begin{array}{l}\text { Estimation using New shrinkage } \\
\text { function }\end{array}$ & 8.8139 & 29.2275 & 13.3936 & 25.5928 & 16.0211 & 24.0369 \\
\hline
\end{tabular}

Table 2: Denoising Results of Fingerprint image using SCAD, hard, Soft and new Thresholding Function: Top Rule

\begin{tabular}{|c|c|c|c|c|c|c|}
\hline & \multicolumn{2}{|c|}{$\sigma=10$} & \multicolumn{2}{|c|}{$\sigma=20$} & \multicolumn{2}{|c|}{$\sigma=30$} \\
\hline & RMSE & PSNR & RMSE & PSNR & RMSE & PSNR \\
\hline Noisy Image & 9.9616 & 28.1642 & 19.9724 & 22.1222 & 29.9724 & 18.5964 \\
\hline Estimation using Soft function & 7.9451 & 30.1288 & 11.2000 & 27.1464 & 14.3554 & 24.9905 \\
\hline Estimation using SCAD function & 8.6454 & 32.3137 & 13.0913 & 25.7911 & 17.7684 & 23.1378 \\
\hline Estimation using Hard function & 8.8571 & 29.1850 & 17.3234 & 23.3581 & 25.4437 & 20.0192 \\
\hline $\begin{array}{l}\text { Estimation using New shrinkage } \\
\text { function }\end{array}$ & 7.2633 & 30.9081 & 11.4354 & 26.9658 & 15.6758 & 24.2262 \\
\hline
\end{tabular}

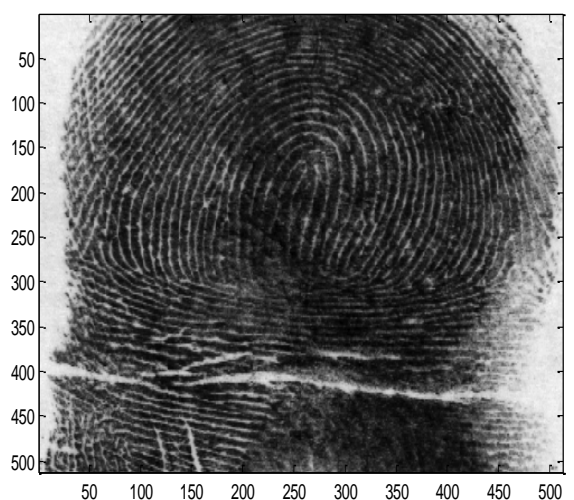

Fig 1: Original image

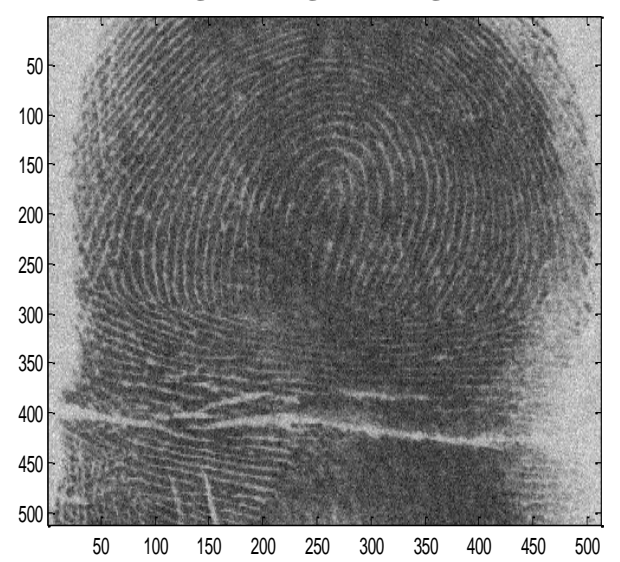

Fig 2: Nosiy image $(\sigma=20)$

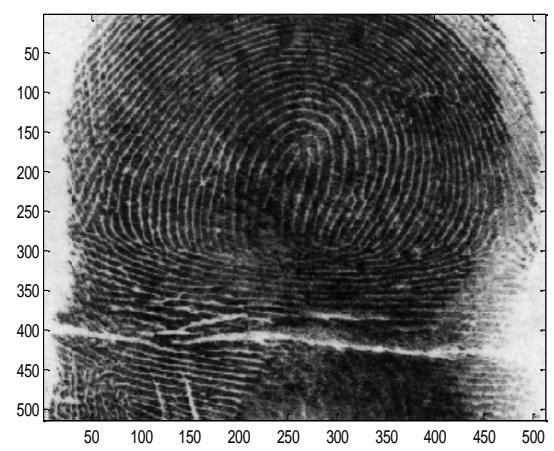

Fig 3: denoised image using new thresholding function with visu rule

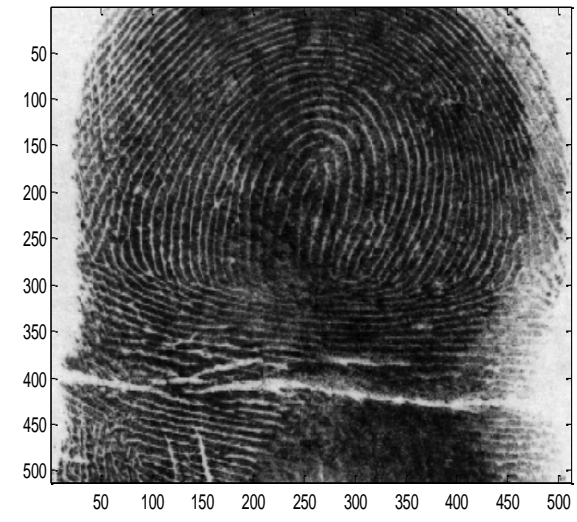

Fig 4: denodied image using new thresholding function with Top rule 


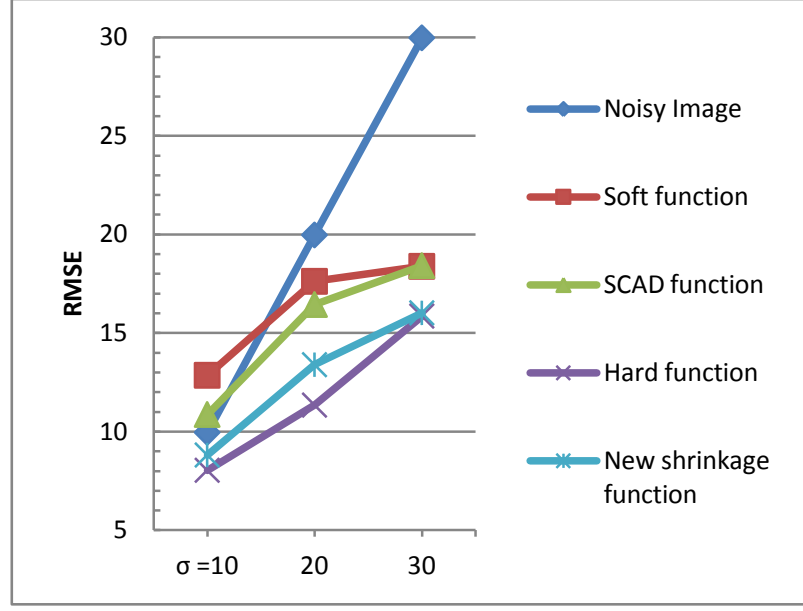

Graph 1: RMSE values of different functions in Visu rule method

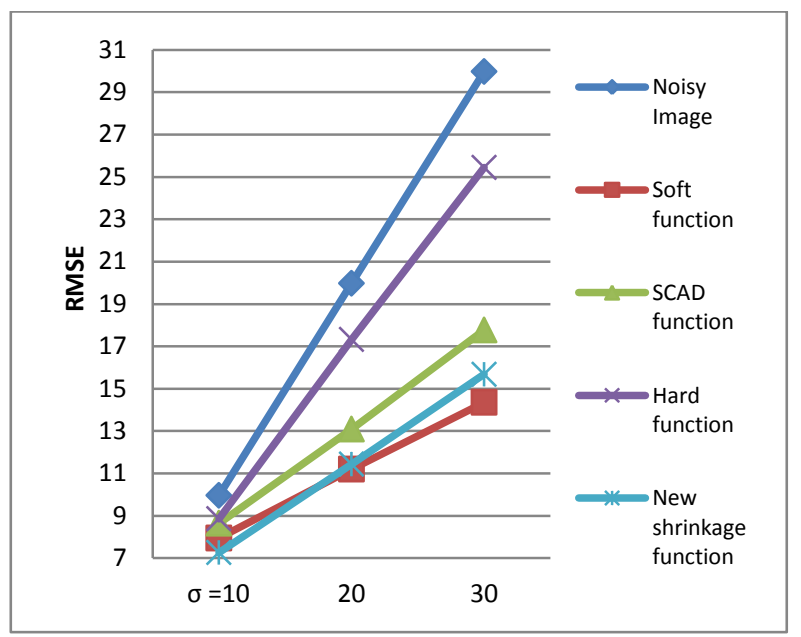

Graph 3: RMSE values of different functions in Top rule method

\section{CONCLUSION}

In this paper, a new thresholding filter for wavelet shrinkage denoising of image is proposed. The performance of this filter is evaluated by using Fingerprint image. The results are compared with existing SCAD, hard and soft filters. It is found that the new thresholding filter performs better than both SCAD and Soft filters with visu rule method and performs better than SCAD and hard function with Top Rule. In this project a new thresholding filter is used for the denoising of black and white images in future it may be able to apply for the colour images and videos also.

\section{ACKNOWLEDGMENTS}

The authors place on record their thanks to the authorities of Amara Institute of Engineering \& Technology College, A.P for the facilities they provided. I would like to express my special gratitude and thanks KOTESWARARAO MALLAPARAPU for giving me such attention and time providing necessary information regarding the project and also for their support in completing the project work.

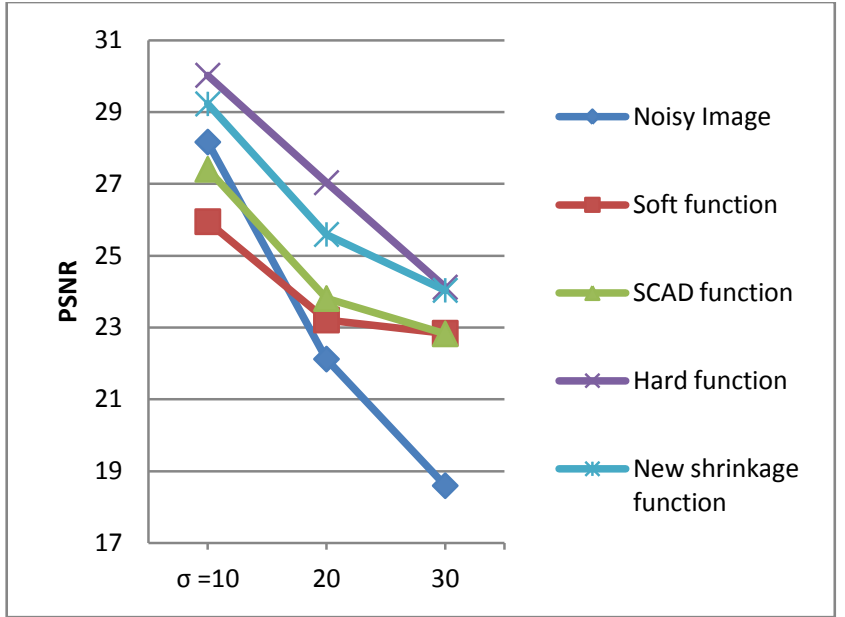

Graph 2: PSNR values of different functions in visu rule method

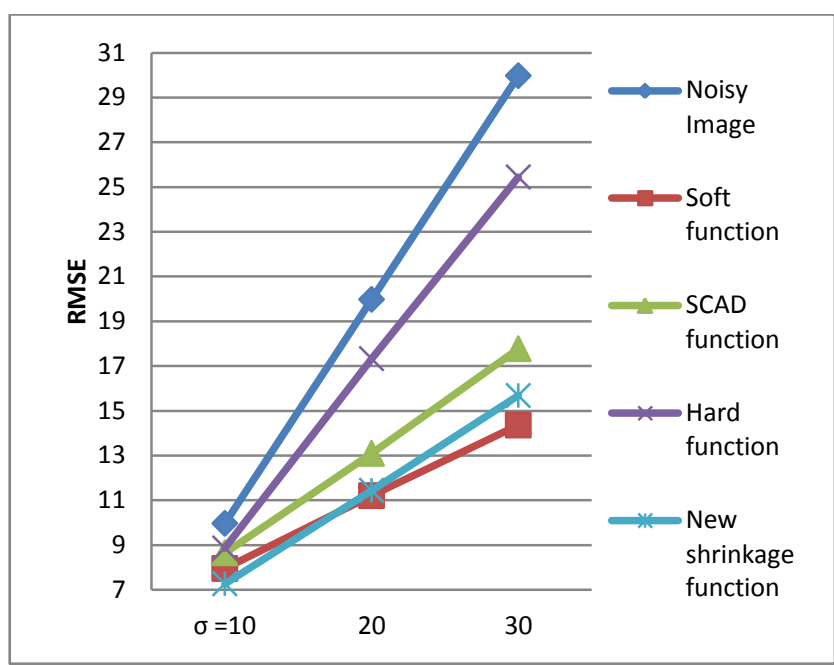

Graph 4: PSNR values of different functions in Top rule method

\section{REFERENCES}

[1] Donoho, D. L., (1995) ,Denoising by Soft Thresholding, IEEE Trans. Information Theory, Vol. 41, No. 3, pp 613 627

[2] Manjit kaur,(2013), image denoising using wavelet transform, International Journal Of Engineering And Computer Science vol. 2, No 10, pp 2932-2935

[3] Sanjay Jangra, Ravinder Nath Rajotiya(2013) An Improved Threshold Value for Image Denoising Using Wavelet Transforms, Sanjay Jangra et al Int. Journal of Engineering Research and Applications ISSN : 22489622, Vol. 3, Issue 6, Nov-Dec 2013, pp.1893-1897

[4] Hari Om, Mantosh Biswas (2012), an Improved Image Denoising Method Based on Wavelet Thresholding, Journal of Signal and Information Processing, vol. 3, pp $109-116$

[5] Dr. V.V.K.D.V.Prasad (2013) 'A New Wavelet Packet Based Method for Denoising of Biological Signals', International Journal of Research in Computer and Communication Technology, Vol.2, Issue.10, pp.10561062. 
[6] KOTESWARARAO M, Dr.V.V.K.D.V.PRASAD, "Decimated and Undecimated Wavelet Transforms Based Estimation of Images" "International Journal of Innovative Research in Science, Engineering and Technology", vol.3, Issue 10, pp 16981-16988,October 2014.

[7] Akhilesh Bijalwan, Aditya Goyal, Nidhi Sethi, (2012) Wavelet Transform Based Image Denoise Using Threshold Approaches, International Journal of Engineering and Advanced Technology (IJEAT) ISSN: 2249 - 8958, Volume-1, Issue-5, June 2012

[8] Ruban Brar, Rajesh Kumar (2013), Image Denoising Using Wavelet Thresholding Hybrid Approach Proceedings of SARC-IRAJ International Conference, 22nd June 2013, New Delhi, India, ISBN: 978-81-
927147-6-9

[9] KOTESWARARAO M, Dr.V.V.K.D.V.PRASAD, "Estimation of Images Using Decimated Wavelet Transform" "International Journal of Image Processing and Applications", vol.5, Issue 2, pp, 223-228, December 2014.

[10] Virendra Kumar, Dr. Ajay Kumar (2013), Simulative analysis for Image Denoising using wavelet thresholding techniques, International Journal of Advanced Research in Computer Engineering \& Technology (IJARCET) Volume 2, No 5, May 2013

[11] Dr. V.V.K.D.V.Prasad (2008) 'Denoising of Biological Signals Using Different Wavelet Based Methods and Their Comparison', Asian Journal of Information Technology, Vol.7, No.4, pp.146-149. 